\title{
Immune response against Treponema spp. and ELISA detection of digital dermatitis
}

\author{
A. Gomez, ${ }^{* 1}$ K. S. Anklam, ${ }^{*}$ N. B. Cook, ${ }^{*}$ J. Rieman, ${ }^{*}$ K. A. Dunbar, ${ }^{*}$ K. E. Cooley, ${ }^{*}$ M. T. Socha, $\dagger$ \\ and D. Döpfer* \\ *School of Veterinary Medicine, University of Wisconsin, Madison 53706-1102 \\ †Zinpro Corporation, 10400 Viking Dr., Ste. 240, Eden Prairie, MN 55374
}

\begin{abstract}
The objective of this longitudinal study was to evaluate the immune response against Treponema spp. infection in dairy heifers affected with digital dermatitis (DD). In addition, the accuracy of an indirect ELISA detecting anti-Treponema IgG antibodies in identifying clinical DD status has been assessed. A cohort of 688 pregnant Holstein heifers was evaluated at least 3 times before calving during a period of 6 mo. Complete clinical assessment of DD presence on the back feet of each heifer and blood extraction were performed in a standup chute. Digital dermatitis cases were characterized by the M-stage classification system and size and level of skin proliferation. An ELISA was performed on blood serum samples obtained from a subcohort of 130 heifers. For description purposes, the animals were classified by the number of clinical cases experienced during the study period as type I (no clinical cases were observed), type II (only 1 acute clinical case diagnosed), and type III (at least 2 acute clinical cases diagnosed). Multivariable repeated-measures models were used to evaluate the immune response against Treponema spp. infection. A binormal Bayesian model for the ELISA data without cut-point values was used to assess the accuracy of the ELISA as a diagnostic tool. Animals that never experienced a DD event throughout the study kept a constant low level of antibody titer. A 56\% increase in mean ELISA titer was observed in heifers upon a first clinical DD case diagnosis. After topical treatment of an acute DD case with oxytetracycline, the antibody titer decreased progressively in type II heifers, achieving mean levels of those observed in healthy cows after a mean of $223 \mathrm{~d}$. Surprisingly, antibody titer was not increased in the presence of M1 (DD lesion $<20 \mathrm{~mm}$ in diameter surrounded by healthy skin) and M4.1 (DD lesion $<20 \mathrm{~mm}$ in diameter embedded in a circumscribed dyskeratotic or proliferative skin altera-
\end{abstract}

Received October 16, 2013.

Accepted April 30, 2014.

${ }^{1}$ Corresponding author: gomez3@wisc.edu tion) DD stages. Type III cows showed a slight increase in antibody levels. The presence of skin proliferation at first DD diagnosis was found to be associated with an odds ratio of 2.04 of becoming a type III heifer in relation to heifers presenting first lesions without skin proliferation. The ELISA validity was estimated by an area under the curve of 0.88 . Predicted probabilities of infection are provided for a range of ELISA values and prevalence of infection. Early detection and treatment is essential to control DD and the ELISA can be used in understanding the immunopathology of DD and shows great promise for prescreening purposes during DD management programs in combination with traditional clinical inspection.

Key words: digital dermatitis, ELISA, Treponema spp., cattle

\section{INTRODUCTION}

Bovine digital dermatitis (DD) is increasingly found worldwide, affecting cattle in all production systems (Hulek et al., 2010; Capion et al., 2012; Chapinal et al., 2013). Footbaths and topical treatments have been extensively used as the main preventive measures to control DD. However, footbathing strategies have obtained variable success (Relun et al., 2013) especially where groups of animals require less frequent handling, such as beef or replacement dairy cattle.

To achieve a "manageable state of disease" (Döpfer and Bonino Morlán, 2008), identifying individual cases of DD as early as possible and acting promptly upon diagnosis is of paramount importance. Clinical inspection of the foot by means of individual restraint is generally recognized as the gold standard diagnostic procedure (Stokes et al., 2012). This method is more labor intensive and time consuming than alternative inspection methods, which are often used given very similar accuracy (Relun et al., 2011; Stokes et al., 2012). Although the different clinical stages of the disease have been clearly described (Döpfer et al., 1997; Berry et al., 2012), subjectivity is present during clinical inspection, which results in the need for specific training to correctly identify clinical DD lesions. 
Evidence supporting the hypothesis that Treponema spp. play a major role in the microbial etiology of the disease (Demirkan et al., 1999; Gomez et al., 2012; Santos et al., 2012) motivated research about alternative indirect diagnostic methods, such as ELISA. It has been extensively reported that different Treponema spp. are present in DD lesions simultaneously (Moe et al., 2010; Rasmussen et al.; 2012 Santos et al., 2012) and crossimmune reaction between different phylotypes favor the hypothesis about common antigenic areas. This crossimmunity allows for the pooling of isolates to generate ELISA assays able to detect a wide range of systemic Treponema spp. responses (Elliott and Alt, 2009; Vink et al., 2009).

Aiming at reducing time and labor and at avoiding subjectivity in the diagnosis process of DD, several authors have developed ELISA with acceptable performance (Vink et al., 2009; Moe et al., 2010, Jones et al. 2012). One of the limitations they faced was having only cross-sectional data available, making it in turn impossible to assess the temporal features of the immune response to treponemes under field conditions. Specifically for dairy heifers or feedlot cattle that are frequently affected by DD (Doherty et al., 1998; Laven and Logue, 2007), and sometimes housed in facilities not adapted to easily handle individual cases to perform clinical inspections, ELISA are attractive to identify DD status. However, incomplete understanding of the immune response to DD (Walker et al., 1997; Trott et al., 2003) has hindered the development of ELISA as a diagnostic tool.

The objective of this longitudinal study was to evaluate the immune response to Treponema spp. in dairy heifers affected with DD and to assess the accuracy of an indirect ELISA in identifying clinical DD status without the need for establishing a fixed cut point for the test.

\section{MATERIALS AND METHODS}

As part of a larger study, a cohort of 688 pregnant heifers was followed for a period of 6 mo [interquartile range $(\mathbf{I Q R})=5.4-6.2]$ in a commercial Holstein dairy herd endemically infected with DD. The heifers entered the study with a mean (SD) days pregnant (DCC) of 75 (18.5) d and a mean age of 17.7 (1.7) mo. The end of the study for each individual animal was defined as the date of moving to a pre-fresh pen at 252 DCC. During the follow-up period, the back feet of each animal were inspected in a stand-up chute (M-Series; Comfort Hoof Care Inc., Baraboo, WI) at enrollment, 3 mo later (IQR $=2.7-3.4 \mathrm{mo}$ ), and on the last day of the follow-up $(\mathrm{IQR}=22.4-24.0 \mathrm{mo})$. Upon detection of a clinical DD lesion, additional evaluations were performed at weekly intervals until clinical cure. Clinical cure was defined as the presence of complete skin restoration or presence of scab tissue covering the lesion, dry appearance, and absence of pain upon manipulation. When a clinically active case was found, topical treatment with $15 \mathrm{~mL}$ of dry tetracycline-HCl powder (Tet-Sol 324; Alpharma Inc., Fort Lee, NJ) was applied directly on the surface of the lesion under a light wrap that was removed between 24 and 48 h. During each visit, DD lesion characteristics (size, presence of proliferation, DD signs, and location), hip height, and heart girth circumference were recorded electronically chuteside for each individual heifer in a Microsoft Access database (Microsoft Corp., Redmond, WA). Lesions were characterized by their size ( $\mathrm{mm}$, measured across the greater dimension), presence of proliferation (4-point scale in ascending severity: "none," "scale," "mass-like," and "filamentous"), presence of heel horn erosion (4-point scale in ascending severity: "none," "diffuse," "v-shaped," and "layered), and location ("heel," "interdigital," "dorsal aspect," or "other location"). Demographic characteristics such as age and DCC, and health events were extracted from the farm software (DC305; Valley Agricultural Software, Tulare, CA; Table 1). In addition, a blood sample was taken from the tail vein at each inspection. The blood samples were processed within the following $12 \mathrm{~h}$ to extract serum and stored at $-80^{\circ} \mathrm{C}$ until the analysis was performed at the completion of the study.

A 5-point scale according to Döpfer et al. (1997) and Berry et al. (2012) was used to classify DD lesions. Namely, lesions were classified as M0 if no lesions compatible with DD were observed at the skin horn border of the foot; M1 and M4.1 if a DD lesion $<20 \mathrm{~mm}$ in diameter was observed surrounded by healthy skin or embedded in a circumscribed dyskeratotic or proliferative skin alteration, respectively, M2 if an active lesion was found with a diameter $\geq 20 \mathrm{~mm}$, and M4 if only a circumscribed dyskeratotic or proliferative skin alteration was identified. Based upon the number of active M2 lesions identified during the follow-up period, all animals were further classified as type I (no M2 lesions identified), type II [only $1 \mathrm{M} 2$ event, allowing the presence of M4 (type II 4) or M4.1 lesions (type II 4.1)], or type III (multiple M2 lesions recorded).

A subcohort of 130 animals falling into one of the type I, II, or III cow categories was randomly selected and their corresponding serum samples were submitted to the laboratory to determine the immune response to Treponema spp. contact by means of an indirect ELISA described below. Specifically, 339, 153, and 209 serum samples corresponding with 66 type I, 34 type II, and 30 type III heifers were processed, respectively. To guarantee a minimum follow-up for each animal, heifers were included in the subcohort when at least 2 evalua- 
Table 1. Demographic characteristics for all heifers at enrollment ${ }^{1}$

\begin{tabular}{|c|c|c|c|c|c|c|c|c|}
\hline Item & \multicolumn{2}{|c|}{ All $(\mathrm{n}=688)$} & \multicolumn{2}{|c|}{ Type I (n = 477) } & \multicolumn{2}{|c|}{ Type II $(\mathrm{n}=84)$} & \multicolumn{2}{|c|}{ Type III $(\mathrm{n}=127)$} \\
\hline Age (mo) & 17.7 & 1.7 & 17.6 & 1.7 & 17.8 & 1.8 & 18.0 & 1.5 \\
\hline Girth (cm) & 185.9 & 8.6 & 184.9 & 6.1 & 185.7 & 7.4 & 188.5 & 8.6 \\
\hline Height $(\mathrm{cm})$ & 139.4 & 4.1 & 138.7 & 4.1 & 139.9 & 3.3 & 140.7 & 4.1 \\
\hline
\end{tabular}

${ }^{1}$ Type I = no M2 (active lesion with a diameter $\geq 20 \mathrm{~mm}$ ) digital dermatitis (DD) cases diagnosed; type II = only $1 \mathrm{M} 2 \mathrm{DD}$ case diagnosed; type III = multiple M2 DD cases diagnosed

${ }^{2} \mathrm{DCC}=$ days of pregnancy.

tions were available. Specifically for type II and type III heifers, at least 4 wk of follow-up after the first M2 DD event were necessary for inclusion in this subcohort.

Missing blood samples due to missing visits were recorded at evaluation $1(\mathrm{n}=21)$, evaluation $2(\mathrm{n}=5)$, and evaluation $3(\mathrm{n}=5)$. Missingness was considered as being random and evaluations with complete information were kept in the data set.

\section{Treponema Antigen Preparation}

Treponeme strains 1-9185MED and 2-1498 isolated from an active DD (Walker et al., 1995) were subcultured in oral treponeme enrichment broth (Anaerobe Systems, Morgan Hills, CA) and $10 \%$ fetal bovine serum (Lonza, Walkersville, MD) and grown in an anaerobic cabinet at $37^{\circ} \mathrm{C}$ for $28 \mathrm{~d}$. Treponemes were harvested by centrifugation at $13,500 \times g$ for $10 \mathrm{~min}$ at $4^{\circ} \mathrm{C}$. The pellet of each strain was washed 3 times in $10 \mathrm{~m} M$ PBS. The final suspension was subjected to 3 cycles of sonication (Sonics \& Materials Inc., Danbury, $\mathrm{CT}$ ) for $2 \mathrm{~min}$ and then a 2 -min rest period in dry ice. The sonicate was centrifuged at $10,000 \times g$ for $30 \mathrm{~min}$ at $4^{\circ} \mathrm{C}$. The protein concentration of the supernatant containing the cellular antigens was determined using a protein assay kit (Thermo Scientific, Rockford, IL) in accordance with the manufacturer's instructions and stored at $-20^{\circ} \mathrm{C}$ until usage. The protein content represents the cellular antigen concentration.

\section{Anti-Treponema Antibody Production}

Bacterial suspensions of Treponema strains 1-9185MED and 2-1498 were supplied to a commercial custom antisera service (Panigen Inc., Blanchardville, WI) for the generation of rabbit IgG antisera. During this procedure, rabbits were immunized in a multisite regimen using Freund's complete and incomplete adjuvants over a 6 -wk period. At the terminal bleed, the IgG antisera were tested for reactivity by ELISA against the antigens of the Treponema strains 1-9185MED and 2-1498 and were shown to cross-react with both strains.
These IgG antisera were then used as the positive controls for the ELISA.

\section{Treponema ELISA}

Standard ELISA methods were used for determination of antibody production. Briefly, 96-well microtiter plates (Nalge Nunc International, Rochester, NY) were coated with $100 \mu \mathrm{L}$ of $5 \mu \mathrm{g} / \mathrm{mL}$ antigens in coating buffer (Kirkegaard \& Perry Laboratories Inc., Gaithersburg, MD) and incubated overnight at $4^{\circ} \mathrm{C}$ (after a preliminary run using 22 duplicate samples using strains 2-1498, 1-9185MED, or a combination of both, only Treponema 2-1498 antigen was used to evaluate the complete sample set due to the higher optical density readings shown by this strain). Unbound antigen was removed by washing 3 times with wash solution (Kirkegaard \& Perry Laboratories Inc.) and the plates were blocked with $300 \mu \mathrm{L}$ of $0.5 \%$ milk diluent/blocking solution (Kirkegaard \& Perry Laboratories Inc.) for $2 \mathrm{~h}$ at room temperature (RT). Sera from cattle were diluted 1:100 in $0.5 \%$ milk diluent-blocking solution and incubated for $1 \mathrm{~h}$ at RT with 60-rpm shaking on a microplate shaker (Labnet International Inc., Edison, NJ). The plates were washed 3 times with wash solution. Diluted sera $(100 \mu \mathrm{L})$ were added to the ELISA plate wells in duplicate and incubated for $1 \mathrm{~h}$ at RT with 60-rpm shaking. Positive (rabbit IgG antisera) and negative (fetal bovine serum) control sera were used in every ELISA plate for each test. The plates were washed 5 times, and then $100 \mu \mathrm{L}$ of protein $\mathrm{G}$ labeled with horseradish peroxidase (Bio-Rad Laboratories Inc., Hercules, CA) diluted 1:5,000 in $10 \mathrm{mM}$ PBS was added as a conjugate, and then incubated for $1 \mathrm{~h}$ at RT with 60-rpm shaking. Following incubation, plates were washed 5 times with wash solution and 100 $\mu \mathrm{L}$ of SureBlue substrate solution (Kirkegaard \& Perry Laboratories Inc.) was added to each well followed by 1 min of incubation at RT. The reaction was stopped by adding $100 \mu \mathrm{L}$ of TMB stop solution (Kirkegaard \& Perry Laboratories Inc.) to each well, and the optical density at $450 \mathrm{~nm}$ was measured with a multiwell ELISA 
plate reader (BioTek Instruments Inc., Winooski, VT). The optical density (OD) values were transformed to sample-to-positive $(\mathbf{S} / \mathbf{P})$ ratios based on the OD for the serum sample along with those for the negative and positive controls, using the following equation: $\mathrm{S} / \mathrm{P}$ ratio $=(\mathrm{OD}$ of sample $-\mathrm{OD}$ of negative control $) /(\mathrm{OD}$ of positive control - OD of negative control).

\section{Statistical Analysis}

Statistical analysis was performed using individual animals as the observational unit. Information from both back legs was summarized for each heifer. Heifers with one or both legs with an M2 lesion were classified as M2, animals with an M4.1 lesion in one or both legs and no M2 lesions were classified as M4.1, animals with an M1 in one or both legs and no M2 or M4.1 lesions were classified as M1, animals with an M4 in one or both legs and no other DD lesion were classified as M4, and animals with no DD lesions were classified as M0. The ELISA S/P ratios were logarithmically transformed [producing log (ELISA S/P) (LESP) values] to facilitate interpretation and to normalize the data.

To predict and analyze the immune response against Treponema spp. infection in animals presenting no disease, M1 or M4.1, and M2 stages, multivariable mixed linear models were used for each case as shown by Equations 1 to 4:

$$
\begin{gathered}
\text { LESP }_{i j} \sim \beta_{0}+\beta_{1} \text { Follow-up time }_{i j}+\beta_{2} \text { Age }_{i j} \\
+\beta_{3} \text { Girth }_{i j} \times \beta_{4} \text { Height }_{i j}+\beta_{5} \text { DCC }_{i j}+\nu_{i}+\varepsilon_{j} ; \\
\text { LESP }_{i j} \sim \beta_{0}+\beta_{1} \text { Follow-up time }_{i j} \times \beta_{2} \text { Sub-DD }_{i j} \\
+\beta_{3} \text { Girth }_{i j} \times \beta_{4} \text { Height }_{i j}+\beta_{5} \mathrm{DCC}_{i j} \\
+\beta_{6} \text { Age }_{i j}+\nu_{i}+\varepsilon_{j} ; \\
\text { LESP }_{i j} \sim \beta_{0}+\beta_{1} \text { Follow-up time }_{i j} \times \beta_{2} \text { BefAfter }_{i j} \\
+\beta_{3} \text { Girth }_{i j} \times \beta_{4} \text { Height }_{i j}+\beta_{5} \mathrm{DCC}_{i j} \\
\quad+\beta_{6} \text { Age }_{i j}+\nu_{i}+\varepsilon_{j} ; \\
\text { LESP }_{i j} \sim \beta_{0}+\beta_{1} \text { Follow-up time }_{i j} \times \beta_{2} \text { Cow type }_{j} \\
+\beta_{3} \text { Girth }_{i j} \times \beta_{4} \text { Height }_{i j}+\beta_{5} \mathrm{DCC}_{i j} \\
+\beta_{6} \text { Age }_{i j}+\nu_{i}+\varepsilon_{j},
\end{gathered}
$$

where $j=j$ th observation of a heifer, $i=i$ th heifer unique identifier, $\nu_{i}=$ autoregressive (1) correlation, and $\varepsilon_{j}=$ residual error; Follow-up time $=$ days from first observation (Equations 1, 2, and 3) or from first
M2 diagnosis (Equation 4), Sub-DD = indicator of M1 or M4.1 DD stages (1), BefAfter = indicator of period of observation before (0) or after (1) the first M2 event, and Cow type $=$ categorical variable including cow type I (referent), type II (4), type II (4.1), and type III, age, girth circumference, height, and DCC at the $j$ th observation of the $i$ th cow.

Dependency between repeated observations for each heifer was accounted for in the models using an autoregressive covariance and the best maximum likelihood fit. Diagnostic plots were checked to assess the assumption of constant variance of the residuals. The relative dependency of the repeated observations was assessed by the intraclass correlation coefficient (ICC). Age, height, weight, and DCC were added to the initial models as fixed effects to correct for those effects and interactions between fixed effects were tested to detect heterogeneity of effects. Significance was set at the 5\% level. Variables were kept in the model if changes in the predictor of interest were greater than 10\%. All final models were selected by the backward elimination method from the basic models (1 to 5 ) and the forward selection method was used when simple or double interactions were tested. The likelihood ratio test to discriminate between nested models and the Akaike information criterion between non-nested models was used to select the more significant parsimonious model.

Due to the epidemiological importance between type II and type III animals and to increase the power of finding different characteristics between DD lesions observed in the 2 groups, a logistic model was applied to the complete data set as shown in Equation 5. Aiming at avoiding confounding factors due to chronicity, only characteristics found at the first M2 lesions record were included in the analysis. This resulted in only 1 observation per animal. Analysis of the deviance of the logistic model was performed using half-normal plots and the $F$-test to compare a dispersion parameter $=1$ against a dispersion parameter calculated using Pearson residuals. Exploratory analysis and implementation of the models were done using $\mathrm{R}$ software ( $\mathrm{R}$ Core Team, 2013).

$$
\begin{gathered}
\operatorname{Logit}(\text { Cow type } \\
) \\
+\beta_{3 i} \operatorname{Girth}_{i} \times \beta_{4}+\beta_{1} \text { Peight }_{i}+\beta_{5} \text { DCC }_{i}+\varepsilon_{i}
\end{gathered}
$$

where $i=i$ th observation at the first M2 DD event and $\varepsilon_{i}=$ residual error of the model; Cow type $=$ Logit [type II (0) or type III (1) heifer indicator], Proliferation = indicator of the existence of proliferation (1) or not (0; referent) at diagnosis, Size $=$ size of the DD lesion across its greatest dimension and age, girth circumference, height, and DCC at the first M2 DD event. 
Table 2. Description of digital dermatitis (DD) lesion characteristics of 130 heifers included in the ELISA cohort $^{1}$

\begin{tabular}{|c|c|c|c|c|c|c|c|}
\hline \multirow[b]{2}{*}{ Item $^{2}$} & \multirow[b]{2}{*}{ Characteristic $^{3}$} & \multicolumn{6}{|c|}{ Cow type ${ }^{4}$} \\
\hline & & No. & $\%$ & No. & $\%$ & No. & $\%$ \\
\hline All evaluations & & 380 & 34 & 310 & 28 & 418 & 37 \\
\hline \multicolumn{8}{|l|}{ Lesion type } \\
\hline M0 & & 340 & 89 & 147 & 47 & 100 & 24 \\
\hline \multirow{4}{*}{ M1 } & HHE & 20 & 83 & 16 & 94 & 32 & 91 \\
\hline & Location & 24 & 100 & 17 & 100 & 30 & 85 \\
\hline & Proliferation & 0 & 0 & 0 & 0 & 1 & 2.9 \\
\hline & Size & 7.2 & 3.6 & 9.6 & 3.9 & 10 & 4.7 \\
\hline \multirow[t]{3}{*}{ M2 } & & - & - & 35 & 11 & 100 & 24 \\
\hline & HHE & - & - & 33 & 93 & 90 & 90 \\
\hline & Location & - & - & 32 & 91 & 95 & 95 \\
\hline \multirow{2}{*}{ M4 } & Proliferation & 0 & 0 & 3 & 4.3 & 10 & 8.7 \\
\hline & Size & 22 & 2.2 & 21.8 & 6.1 & 24.5 & 7.7 \\
\hline \multirow[t]{5}{*}{ M4.1 } & & 4 & 1.1 & 40 & 13 & 69 & 16 \\
\hline & HHE & 3 & 75 & 37 & 92 & 64 & 92 \\
\hline & Location & 4 & 100 & 40 & 100 & 69 & 100 \\
\hline & Proliferation & 0 & 0 & 1 & 2.5 & 4 & 5.8 \\
\hline & Size & 19 & 8.8 & 21.0 & 4.1 & 24.6 & 6.0 \\
\hline
\end{tabular}

${ }^{1}$ All observations are included. The number of evaluations for each heifer ranged from 2 to 10 .

${ }^{2} \mathrm{M} 0=$ no DD lesion; M1 = DD lesion $<20 \mathrm{~mm}$ in diameter observed surrounded by healthy skin; M2 = active lesion with a diameter $\geq 20$ mm; $\mathrm{M} 4$ = only a circumscribed dyskeratotic or proliferative skin alteration; M4.1 = digital dermatitis lesion $<20 \mathrm{~mm}$ in diameter embedded in a circumscribed dyskeratotic or proliferative skin alteration.

${ }^{3} \mathrm{HHE}=$ heel horn erosion lesions presenting scores 3 or 4 (v-shaped, layered); location $=$ lesions present in the heel area; proliferation $=$ lesions presenting scores of 3 or 4 (mass-like, filamentous); size = mean and SD.

${ }^{4}$ Type I = no M2 DD cases diagnosed; type II = only 1 M2 DD case diagnosed (allowing the presence of an M4 or M4.1 lesion); type III = multiple M2 DD cases diagnosed.

With the objective of evaluating the accuracy of the ELISA as a diagnostic tool to detect animals currently having an M2 DD case or having had one during the last $150 \mathrm{~d}$, statistical analysis was performed using a binormal Bayesian model as described in Christensen et al. (2011). Receiver operating characteristic curves, area under the curve (AUC), and posterior predictive probability of disease were calculated using clinical inspection as a gold standard. To maximize the efficiency of the information at hand, no cut points were initially established, given that ELISA measurements were obtained on a continuous scale. Pointwise posterior bands and confidence intervals were calculated at $95 \%$. The ELISA S/P ratios from M0 animals were used as control data and data from animals having at least one M2 event were used as the comparison group. The final models were run using 10,000 iterations. Uninformative prior distributions were applied and modifications of the initial values were used to check the robustness of the model. Convergence was achieved in all cases. Models were implemented in the OpenBUGS software (Lunn et al., 2009).

\section{RESULTS}

\section{Patterns of Immune Response}

To describe the response observed over time to Treponema spp. infection, ELISA results are separately summarized for animals showing no disease, M1 or M4.1 and M2 DD. For all evaluations, DD lesion characteristics are shown in Table 2.

No $\boldsymbol{D} \boldsymbol{D}$. Serum samples $(\mathrm{n}=260)$ from 41 animals with no DD events diagnosed during the follow-up time showed a mean (95\% CI) LESP value of $-2.25(-2.44$ to -2.05$)$. No significant increase in LESP values $(0.07$ every $100 \mathrm{~d}$; $P=0.051$ ) was observed during the followup time, estimated by a multivariable model (model 1) using an autoregressive covariance with an ICC of $75 \%$. The observed pattern during the follow-up time was not significantly modified by age, weight, height, or DCC (Figure 1).

M1 or M4.1 DD. Serum samples $(\mathrm{n}=79)$ from 25 animals diagnosed with at least 1 DD M1 or M4.1 and no M2 events during the follow-up period did not 


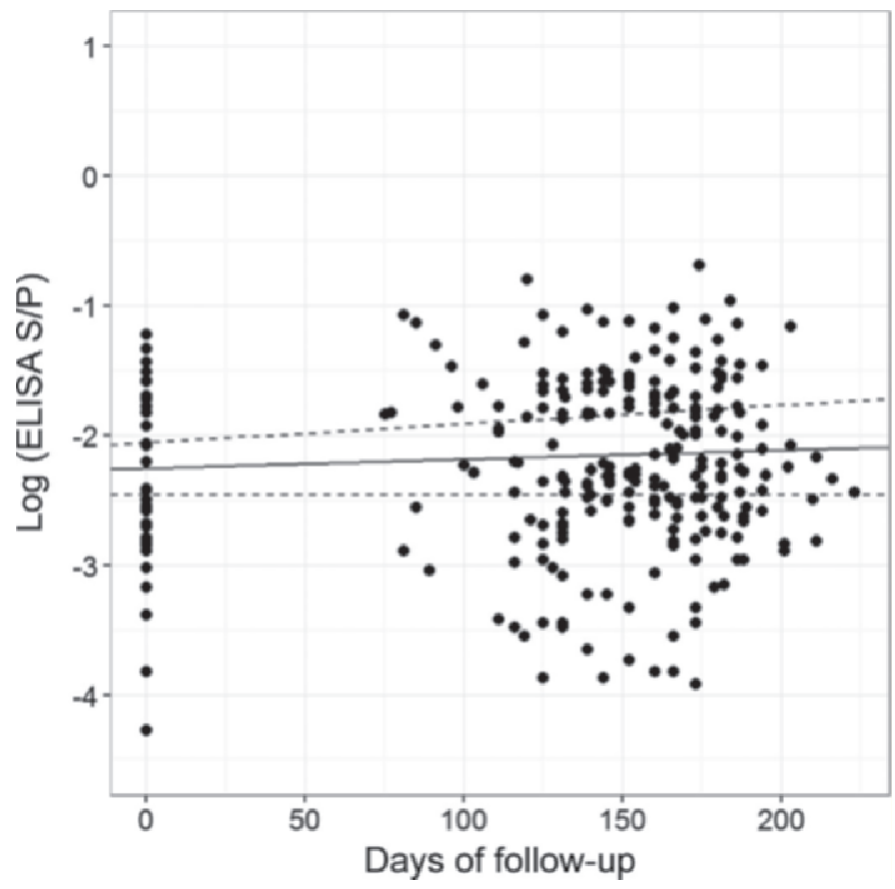

Figure 1. Logarithmically transformed ELISA sample-to-positive $(\mathrm{S} / \mathrm{P})$ ratios $[\log ($ ELISA S/P) (LESP) values] observed during the follow-up time in heifers with no digital dermatitis (DD) events diagnosed (type I; solid line; mean predicted values model 1, including SEM).

show an increment in antibody titer when an M1 or M4.1 DD stages event were identified (Figure 2). Mean (SD) LESP values for M1 or M4.1 DD stages and no DD observations were $-2.17(0.56)$ and $-2.33(0.37)$ respectively. The multivariable model (model 2) fit using an autoregressive covariance showed no statistically significant difference in mean $(95 \% \mathrm{CI})$ LESP values over time $[0.048(-0.128$ to 0.224$)]$ and an ICC of $42 \%$. The difference was not significantly modified by age, weight, height, or DCC (Figure 2).

M2 $\boldsymbol{D D}$. Serum samples $(\mathrm{n}=362)$ from 64 animals diagnosed with at least a clinical (M2) DD event during the follow-up period were used to describe the humoral response against Treponema spp. Unadjusted mean (SD) LESP values for M0, M1, M2, M4, and M4.1 cows were -2.05 (0.78), $-1.78(0.75),-0.94(0.71),-1.12$ $(0.77)$, and $-1.16(0.65)$, respectively. The multivariable model (model 3), fit using an autoregressive covariance and allowing the slope to vary before and after M2 diagnosis, showed a significant increase in mean (95\% CI) LESP values of 0.45 (0.16 to 0.73$)$ in heifers diagnosed at their first M2 event (Figure 3) and the model had an ICC of $31 \%$. The difference in slopes of the regression lines (before and after the first M2 DD diagnosis) and the time of observation was also found to be significantly different $(P<0.01)$.
A different immune response pattern was observed between type II (single M2 event) and type III (multiple M2 events) heifers (model 4) after diagnosis of the first M2 event. Antibody values were seen to decrease over time in type II animals, independent of any subsequent M1 or M4.1 DD stage event. In contrast, in type III cows, LESP values increased steadily (Figure 4). This difference in immune response observed over time was significant (Table 3). In type II cows, LESP values were predicted to return to the mean LESP value observed in type I cows $(-2.23)$ in a period of 216 (188 to 252$) \mathrm{d}$.

Type II and type III heifers showed proliferative M2 lesions upon first recording in 13.2 and $29.9 \%$ of cases, and 186 (49) d and 139 (43) d mean (SD) pregnancy, respectively. In the univariate analysis, the level of skin proliferation on first M2 lesions showed a significant association with the classification of type II and type III cows [mean (95\% CI) OR = 2.04 (1.4 to 2.6); model 5]. When all covariates were included in the logistic model, a significant association $(P<0.001)$ with DCC and a trend in the association with the level of skin proliferation $(P=0.07)$ at first M2 diagnosis was observed. The estimated mean $(95 \% \mathrm{CI})$ odds of becoming a type III heifer was 2.06 (0.94 to 4.68) more in heifers diagnosed with a proliferative first M2 lesion compared with heifers presenting M2 lesions without proliferation. Similarly, increased odds of becoming a type III animal was

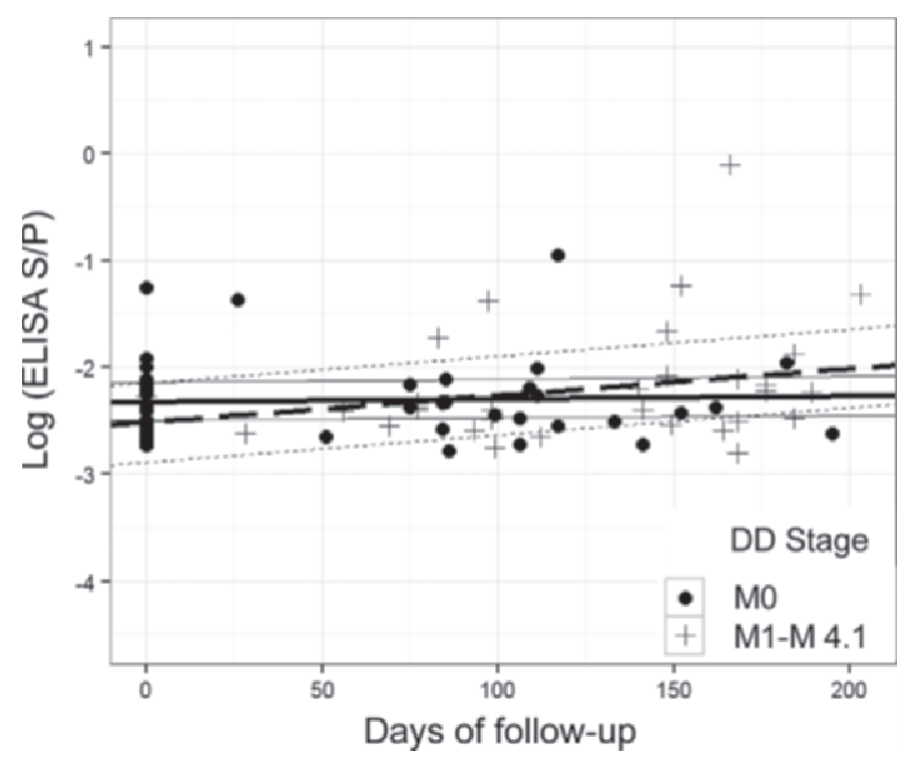

Figure 2. Logarithmically transformed ELISA sample-to-positive $(\mathrm{S} / \mathrm{P})$ ratios $[\log ($ ELISA S/P) (LESP) values] observed during the follow-up time for observations corresponding to no digital dermatitis [DD; M0; $\bullet$ and solid line (mean and 95\% CI)] or M1 and M4.1 DD stage $[+$ and dashed line (mean predicted values of model 2 and $95 \%$ $\mathrm{CI}$ ] events in type I (no DD events diagnosed) cows, where M1 and M4.1 correspond to a DD lesion $<20 \mathrm{~mm}$ in diameter observed surrounded by healthy skin or embedded in a circumscribed dyskeratotic or proliferative skin alteration, respectively. 


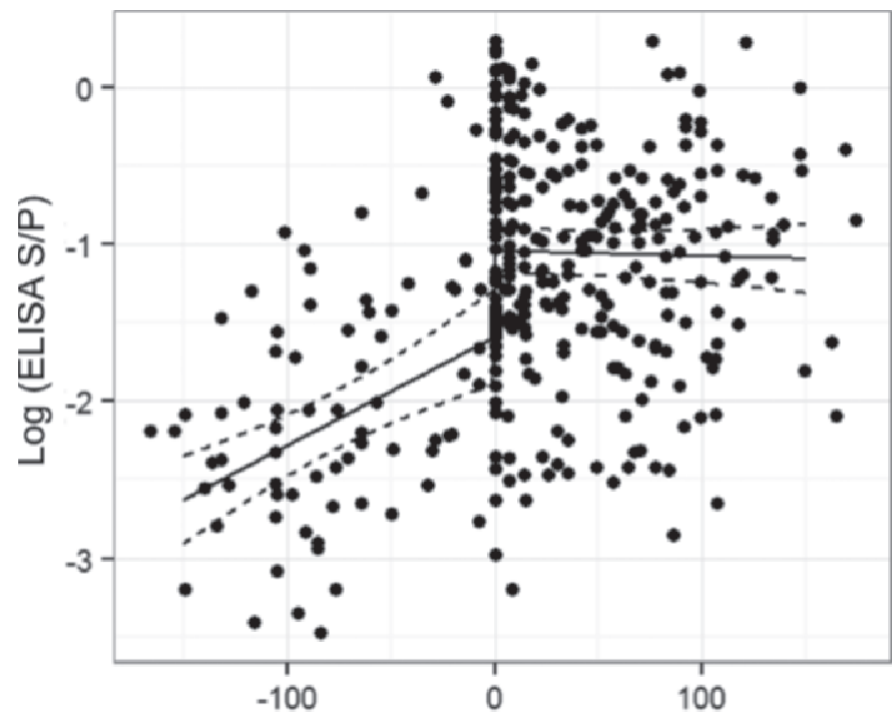

Days around first M2 lesion

Figure 3. Logarithmically transformed ELISA sample-to-positive $(\mathrm{S} / \mathrm{P})$ ratios $[\log ($ ELISA S/P) (LESP) values] observed during the follow-up time for observations corresponding to heifers diagnosed with at least 1 M2 digital dermatitis (DD) event (solid line; mean predicted values of model 3 and 95\% CI), where M2 corresponds to an active lesion with a diameter $>20 \mathrm{~mm}$. The $\mathrm{x}$-axis is zeroed at the first M2 diagnosis. associated with earlier pregnancy stages, showing an $\mathrm{OR}=0.81$ (0.80 to 0.815$)$ for every $10 \mathrm{~d}$ less DCC.

\section{ELISA Accuracy and Predictive Probability of Disease}

Output from the binormal Bayesian model showed a mean (95\% CI) AUC of 0.89 (0.84 to 0.93 ) and 0.88 (0.85 to 0.90) when LESP values at the first M2 event or when any DD event during the subsequent $150 \mathrm{~d}$ of follow-up were considered respectively in the analysis (Figure 5). No significant difference between the curves was found. Plots showing the mean (95\% CI) probability of infection simulated using the Markov chain procedures are displayed in Figure 6 for a DD prevalence of 5 and $50 \%$. Table 4 summarizes predicted LESP cut points for a wide range of observed prior prevalence of infection and predicted probabilities of DD infection of 5 to $20 \%$.

\section{DISCUSSION}

\section{Patterns of Immune Response}

In agreement with previously published research, clinical DD cases were found to be consistently as- a)

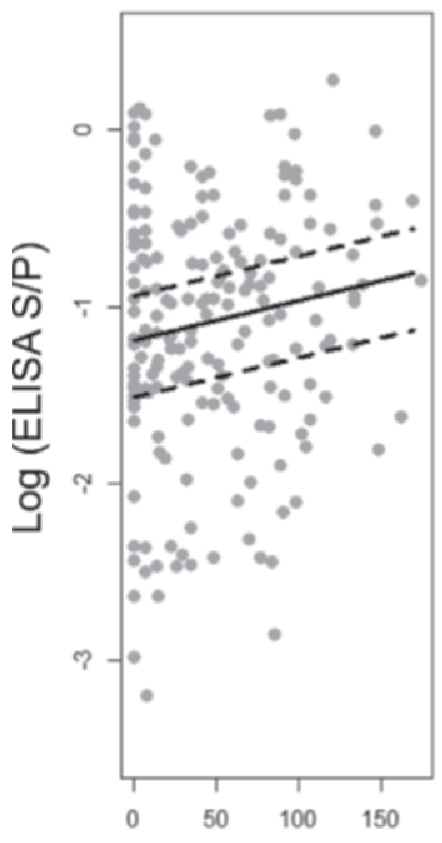

b)

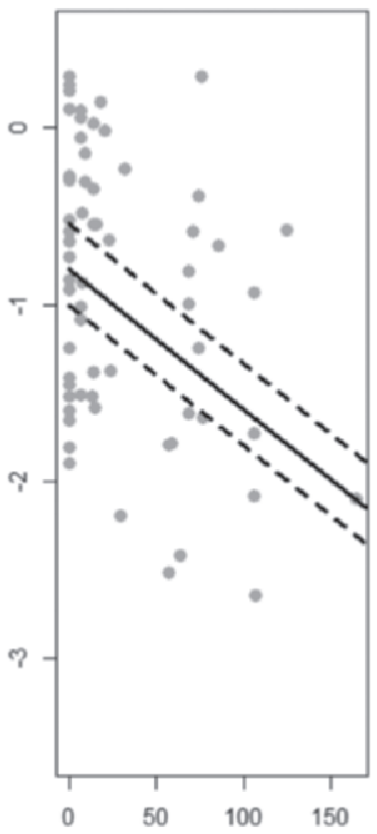

c)

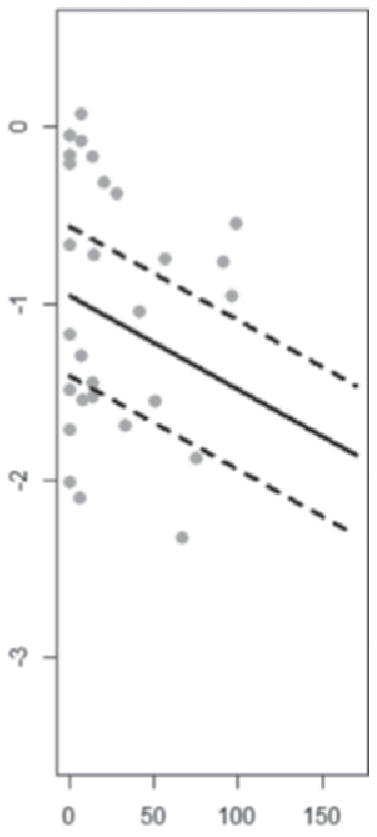

Days around first M2 lesion

Figure 4. Logarithmically transformed ELISA sample-to-positive (S/P) ratios [log (ELISA S/P) (LESP) values] observed over time (dots) and predicted (95\% CI) mean values (lines) from the multivariable model 4 in (a) type III [multiple M2 lesions (active lesions with a diameter $>20 \mathrm{~mm}$ ) recorded], (b) type II 4 [only $1 \mathrm{M} 2$ event, with the presence of an M4 (only a circumscribed dyskeratotic or proliferative skin alteration) lesion], and (c) type II 4.1 heifers [only $1 \mathrm{M} 2$ event, with the presence of an M4.1 lesion (digital dermatitis lesion <20 mm in diameter embedded in a circumscribed dyskeratotic or proliferative skin alteration)]. 
Table 3. Summary of the final multivariable model (model 4), showing the change in logarithmically transformed ELISA sample-to-positive (S/P) ratios [log (ELISA S/P) (LESP) values] after diagnosis of the first M2 (active lesion with a diameter $\geq 20 \mathrm{~mm}$ ) event (intraclass correlation coefficient $=31 \%$ )

\begin{tabular}{lccccc}
\hline Item & Estimate & SEM & $95 \%$ CI & df & $P$-value \\
\hline Intercept & -0.796 & 0.149 & -1.091 to -0.502 & 213 & $<0.01$ \\
Cow type $^{2}$ & Referent & - & - & 56 & 0.13 \\
Type II (4) & -0.154 & 0.277 & -0.712 to 0.039 & & \\
Type II (4.1) & -0.393 & 0.189 & -0.759 to -0.003 & & \\
Type III & -0.008 & 0.001 & -0.010 to -0.004 & 213 & \\
DFM2 & Referent & - & - & 213 & $<0.01$ \\
DFM2: cow type & 0.002 & 0.003 & -0.003 to 0.008 & & \\
DFM2: type II (4) & 0.010 & 0.001 & 0.006 to 0.013 & & \\
DFM2: type II (4.1) & DFM2: type III & &
\end{tabular}

${ }^{1}$ Type III [multiple M2 digital dermatitis (DD) cases diagnosed] $P$-values.

${ }^{2}$ Type II $(4)=$ heifers with a single M2 event and no M1 (DD lesion $<20 \mathrm{~mm}$ in diameter observed surrounded by healthy skin) or M4.1 (lesion $<20 \mathrm{~mm}$ in diameter embedded in a circumscribed dyskeratotic or proliferative skin alteration) DD events diagnosed during the follow-up period; type II (4.1) = heifers with a single M2 event and at least $1 \mathrm{M} 1$ or M4.1 DD event diagnosed during the follow-up period.

${ }^{3} \mathrm{DFM} 2=$ days of follow-up since the first M2 diagnosis.

sociated with a positive humoral immune response to Treponema spp. infection (Demirkan et al., 1999; Moe et al., 2010). Throughout the study, heifers never diagnosed with DD lesions kept constant levels of antiTreponema titer, indicating that although detectable levels of immune response can be observed in endemically infected herds (Walker et al., 1997), lack of clinical disease is consistent with a significantly lower and constant response over time (Figure 1). Furthermore, a dramatic $56 \%$ increase in antibodies was observed

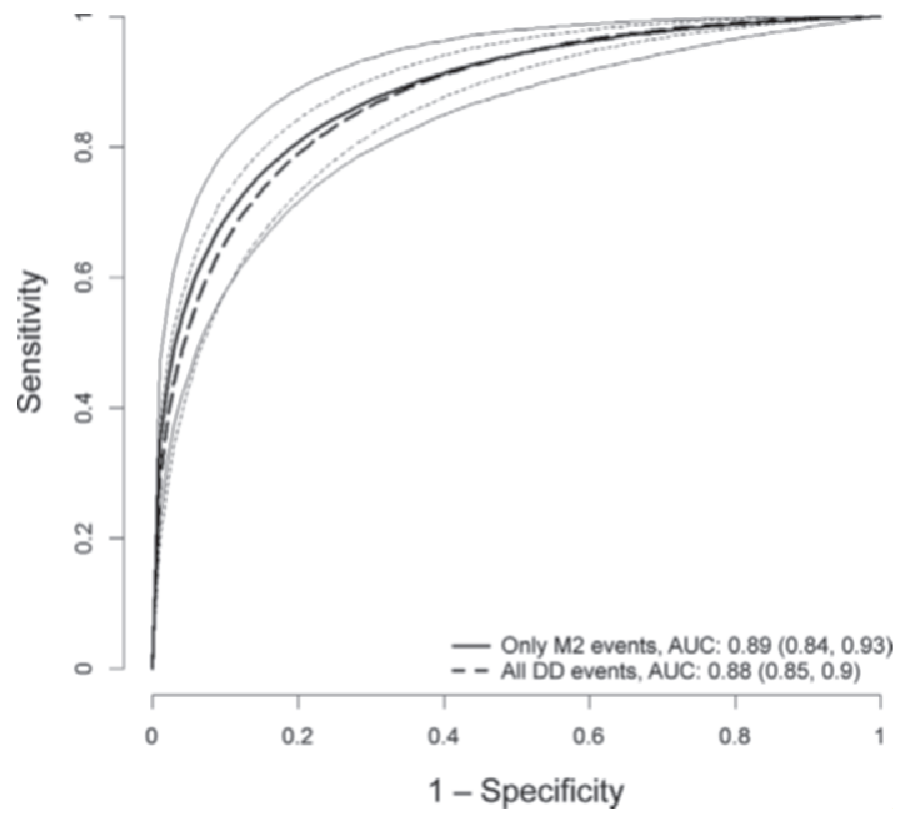

Figure 5. Receiver operating characteristic curves (mean and $95 \%$ CI) for ELISA determination of digital dermatitis (DD) status in heifers at M2 (active lesion with a diameter $\geq 20 \mathrm{~mm}$ ) diagnosis only (dashed line) or at any evaluation during $150 \mathrm{~d}$ after the first M2 diagnosis (solid line). AUC = area under the curve. in heifers upon diagnosis of an M2 stage (Figure 3), reflecting a clear implication of Treponema spp. organisms in the pathogenesis of clinical DD, as extensively reported in the literature (Evans et al., 2009; Capion et al., 2012; Santos et al., 2012). This hypothesis is supported by the fact that heifers experiencing repeated M2 DD cases (type III) maintained elevated titer over time (Figure 4a; Table 3), whereas a gradual decrease in IgG antibodies was found in heifers diagnosed with a non-relapsed DD M2 (type II) case (Table 3).

Cellular immune response previously reported by Trott et al. (2003) had showed a decrease of spirochetespecific lymphocyte blastogenic memory after a period of $42 \mathrm{~d}$. More poorly documented is the humoral response over time. In the present study, it was shown that when no DD occurred, a period of about 6 mo was needed for the antibodies to come back to the mean level value of those observed in healthy heifers.

Contrary to expectations, antibody levels were not significantly increased upon diagnosis of M1 or M4.1 DD stages in animals never experiencing an M2 case during the study (Figure 2) or in animals after being diagnosed with an M2 event (Table 3). These results could be due to a time-lag IgG response identifiable by ELISA and the clinical observation of M1 or M4.1 stages of the disease. Additionally, the occurrence of only a detectable response in clinical DD cases might suggest a dose-dependent response in relation to the total number of spirochetes or a dependency on the penetration of the microorganisms into deeper layers of the skin to boost the immune system (Read and Walker, 1998). Possible interactions between tetracycline treatment and the ELISA detection of serological response to DD infection have not been reported in the literature. 
Table 4. Mean logarithmically transformed ELISA sample-to-positive (S/P) ratios [log (ELISA S/P) (LESP) values] and 95\% CI (in parentheses), corresponding to an arbitrary probability of infection given a determined prior prevalence

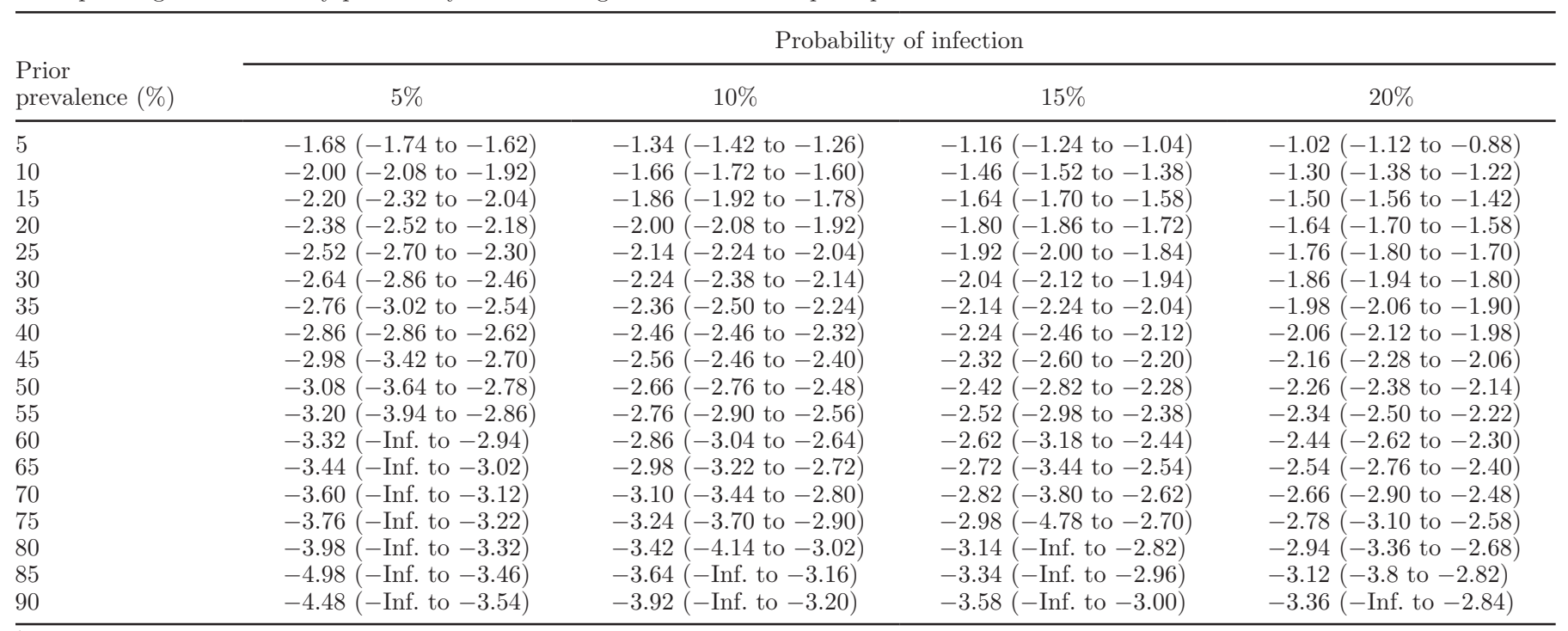

${ }^{1}$-Inf. $=$ lower confidence limit is not defined.

Nonsignificantly different LESP values were observed over time in type II cows when they were dichotomized as heifers with and without M4.1 events after the first $\mathrm{M} 2$ case (Figure 4b and 4c). Incomplete treatment success might favor the progression of spiral Treponema spp. forms to become encysted as described in Umemoto et al. (1984) and Döpfer et al. (2012). Nothing is yet known about the systemic recognition of the presence of Treponema spp. in their encysted forms in cattle but we hypothesize that these forms could remain undetected by the immune system in deep epidermal layers until favorable conditions would stimulate regrowth. Similarly, equivalent forms of resistance have been described in other spirochetal diseases such as syphilis (Brorson et al., 2009) or Lyme disease (Miklossy et al., 2008).

Interestingly, differences in lesion characteristics between type II and type III heifers were found to be significantly associated with skin proliferation upon first M2 lesion diagnosis. Proliferative stages of DD have been traditionally identified in later stages of the disease
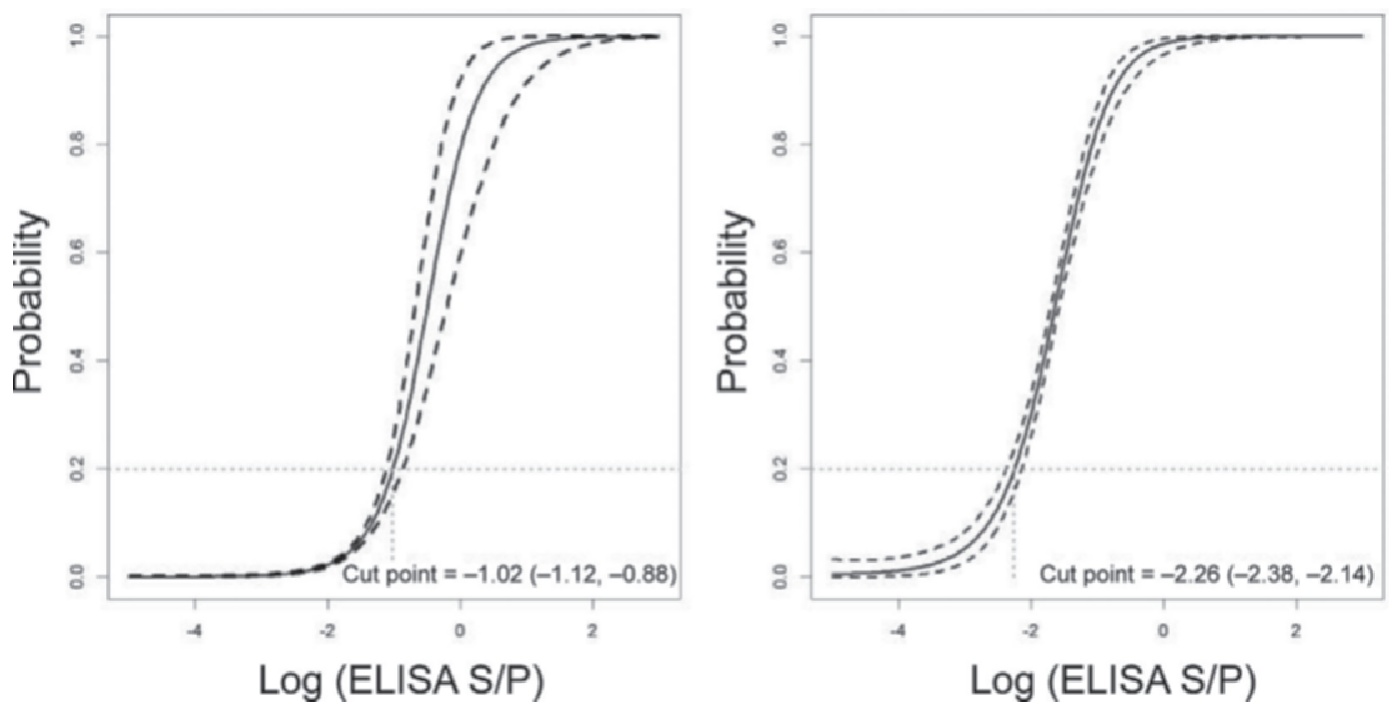

Figure 6. Predicted probability of infection (solid lines) and 95\% CI (dashed lines) for a given digital dermatitis (DD) prevalence given the logarithmically transformed ELISA sample-to-positive (S/P) ratio [log (ELISA S/P) (LESP) value] observed. As an example, only plots for a 5 and $50 \%$ prior prevalence of infection and cut points $(95 \% \mathrm{CI})$ for a $20 \%$ predicted probability of infection are shown. 
(Read and Walker, 1998; Greenough, 2007); thus, DD lesions with increased signs of chronicity are thought to be more prone to relapse after treatment than lesions presenting ulcerations without skin hyperkeratosis. The main reasons might be that, first, morphological changes in the foot conformation occurring shortly after disease onset can modify the local environmental conditions of the foot, leading to the aggravation and progression of DD (Gomez et al., 2013), and second, the presence of hyperkeratotic skin layers covering the lesion might impede topical treatment agents to reach the deeper layers of the skin, which results in failure to reach the bacterial population located deep in the skin (Read and Walker, 1998; Berry et al., 2012). In addition, treatment could hypothetically stimulate active treponemal forms to become encysted due to exposure to suboptimal growth conditions, as reported in Brorson et al. (2009) and Döpfer et al. (2012).

It was clear throughout the study that prompt topical treatment upon early diagnosis led to a decreased percentage of lesions relapsing after a clinical cure and to a smaller number of heifers transitioning from type II to type III. Potential efficacy differences between the treatments available in the market for treating DD lesions could also influence the rates of transition from type II to type III.

\section{ELISA Accuracy and Predictive Probability of Disease}

Development of the ELISA as a Diagnostic Test. Cross-reactivity between Treponema spp. organisms has been well documented (Elliott et al., 2007; Vink et al., 2009). Earlier ELISA studies aiming at identifying animals infected with Treponema spp. organisms have demonstrated the adequacy of using multi-phylotype cocktails of antibodies to improve diagnosis (Moe et al., 2010). In the present study, the approach was using only a monoclonal culture of Treponema phagedenis-like bacteria. The objective was to obtain a valid procedure, given the existence of cross-reactivity, and to simplify the assay. As reported previously in Demirkan et al. (1999) and Elliott et al. (2007), we also aimed to detect IgG response.

The ELISA performed extremely well, discriminating between heifers experiencing an acute DD event and heifers not diagnosed with DD (Figure 5). Median AUC of 0.89 and 0.88 for detection of acute M2 cases or detection of any DD event, respectively, are comparable to other alternative methods, such as DD detection in the milking parlor (Laven, 1999; Relun et al., 2011). Practical implications can be drawn from this fact: (1) the ELISA offers a valid method to evaluate the immune response over time against DD and to increase the understanding of its pathophysiology, (2) the ELISA offers an alternative to clinical inspection in detecting acute clinical M2 cases when labor or trained personnel is limited, and (3) the relatively slow decay in antibodies after an M2 diagnosis allows the ELISA to identify heifers that have had an M2 DD case during the last $150 \mathrm{~d}$, easily missed during visual inspection given the normalization of the lesion physical appearance after treatment.

Bayesian Model Implementation. Clinical inspection performed in the chute under leg restraint has been reported as the gold standard method to diagnose DD in the literature (Stokes et al., 2012). In addition, some authors indicated a good method to ascertain infection status to be a combination between histopathology and clinical inspection (Read and Walker, 1998; Capion et al. 2012). Given that the use of histopathology to support the clinical inspection is invasive and interferes with the natural progression of the disease, it was unfeasible in the present study and clinical inspection alone was used as a gold standard.

To use information obtained from the ELISA more efficiently, no cut-point values were established. As ELISA values were obtained on a continuous scale, using a binormal Bayesian model was aimed at obtaining posterior means for 2 purposes: (1) obtain the predictive probability of DD disease for a particular individual and (2) estimate the proportion of diseased individuals in the population having a given test outcome (Christensen et al., 2011).

In general, DD is an endemic disease on farms and it is controlled when the "manageable state of disease" is reached (Döpfer and Bonino Morlán, 2008). The "manageable state of disease" is defined when the DD prevalence present in the farm makes the likelihood of an outbreak small and the economic consequences due to DD is balanced with the cost of DD prevention control. Thus, depending on the characteristics of the farm or on a particular group of animals, the intensity of claw health management programs might target different levels of DD prevalence. Consequently, the use of predicted probabilities rather than a single fixed cut point to determine DD status offers more flexibility. In this way, less intense DD control programs allow a certain level of infection before treatment or intervention, whereas aggressive programs will reject even very low probabilities of having M2 DD. To serve as a guideline across a range of predicted probabilities of $\mathrm{DD}$ given a specific prior prevalence of disease, cut-point LESP values are summarized in Table 4 as an example. If the information about the prevalence of the DD in a group of animals under evaluation is available, different cutpoint LESP values can then be used in the decisionmaking process of selecting these animals for further 
evaluation. Clear benefit of using an ELISA as a tool can be seen in dairy heifers and beef steers significantly affected by DD (Laven and Logue, 2007; Hulek et al. 2010), where individual inspection of the feet of the animals may be challenging.

\section{CONCLUSIONS}

It is crucial to understand the longitudinal immune response against Treponema spp. infection to take a step forward in managing DD. The presence of layers of proliferative skin on DD lesions could have major implications for how the disease becomes and stays endemic in modern dairy operations. Early detection and treatment are key to control DD. An ELISA can be used for understanding the immunopathology of DD and as an additional tool in DD management programs when used in combination with traditional clinical inspection.

\section{ACKNOWLEDGMENTS}

This study was supported by Zinpro Corporation (Eden Prairie, MN).

\section{REFERENCES}

Berry, S. L., D. H. Read, T. R. Famula, A. Mongini, and D. Döpfer. 2012. Long-term observations on the dynamics of bovine digital dermatitis lesions on a California dairy after topical treatment with lincomycin-HCl. Vet. J. 193:654-658.

Brorson, Ø., S. H. Brorson, J. Scythes, J. MacAllister, A. Wier, and L. Margulis. 2009. Destruction of spirochete Borrelia burgdorferi round-body propagules (RBs) by the antibiotic tigecycline. Proc. Natl. Acad. Sci. USA 106:18656-18661.

Capion, N., M. Boye, C. T. Ekstrøm, and T. K. Jensen. 2012. Infection dynamics of digital dermatitis in first-lactation Holstein cows in an infected herd. J. Dairy Sci. 95:6457-6464.

Chapinal, N., A. Koeck, A. Sewalem, D. F. Kelton, S. Mason, G. Cramer, and F. Miglior. 2013. Genetic parameters for hoof lesions and their relationship with feet and leg traits in Canadian Holstein cows. J. Dairy Sci. 96:2596-2604.

Christensen, R., W. O. Johnson, A. J. Branscum, and T. E. Hanson. 2011. Bayesian Ideas and Data analysis. An introduction for Scientists and Statisticians. Chapman and Hall/CRC Press, Boca Raton, FL. Accessed May 30, 2014. http://www.ics.uci. edu/ wjohnson/BIDA/BIDABook.html.

Demirkan, I., R. L. Walker, R. D. Murray, R. W. Blowey, and S. D. Carter. 1999. Serological evidence of spirochaetal infections associated with digital dermatitis in dairy cattle. Vet. J. 157:69-77.

Doherty, M. L., H. F. Bassett, B. Markey, A. M. Healy, and D. Sammin. 1998. Severe foot lameness in cattle associated with invasive spirochaetes. Ir. Vet. J. 51:195-198.

Döpfer, D., K. Anklam, D. Mikheil, and P. Ladell. 2012. Growth curves and morphology of three Treponema subtypes isolated from digital dermatitis in cattle. Vet. J. 193:685-693.

Döpfer, D., A. Koopmans, F. A. Meijer, I. Szakáll, Y. H. Schukken, W. Klee, R. B. Bosma, J. L. Cornelisse, A. J. A. M. van Asten, and A. A. H. M. ter Huurne. 1997. Histological and bacteriological evaluation of digital dermatitis in cattle, with special reference to spirochaetes and Campylobacter faecalis. Vet. Rec. 140:620-623.

Döpfer, D., and J. Bonino Morlán. 2008. The paradox of modern animal husbandry and lameness. Vet. J. 175:153-154.

Elliott, M. K., and D. P. Alt. 2009. Bovine immune response to papillomatous digital dermatitis (PDD)-associated spirochetes is skewed in isolate reactivity and subclass elicitation. Vet. Immunol. Immunopathol. 130:256-261.

Elliott, M. K., D. P. Alt, and R. L. Zuerner. 2007. Lesion formation and antibody response induced by papillomatous digital dermatitis-associated spirochetes in a murine abscess model. Infect. Immun. 75:4400-4408.

Evans, N. J., J. M. Brown, I. Demirkan, P. Singh, B. Getty, D. Timofte, W. D. Vink, R. D. Murray, R. W. Blowey, R. J. Birtles, C. A. Hart, and S. D. Carter. 2009. Association of unique, isolated treponemes with bovine digital dermatitis lesions. J. Clin. Microbiol. 47:689-696.

Gomez, A., N. B. Cook, N. D. Bernardoni, J. Rieman, A. F. Dusick, R. Hartshorn, M. T. Socha, D. H. Read, and D. Döpfer. 2012. An experimental infection model to induce digital dermatitis infection in cattle. J. Dairy Sci. 95:1821-1830.

Gomez, A., N. B. Cook, K. Cooley, K. Dunbar, J. Rieman, and D. Döpfer. 2013. Is digital dermatitis a cause of hoof conformation changes in its early clinical stage? Page 162 in Proc. Am. Assoc. Bov. Pract., Milwaukee, WI. VM Publishing, Stillwater, OK.

Greenough, P. R. 2007. Bovine Laminitis and Lameness: A Hands-On Approach. 1st ed. Pages 208-211. Saunders Elsevier Ltd., Philadelphia, PA.

Hulek, M., I. Sommerfeld-Stur, and J. Kofler. 2010. Prevalence of digital dermatitis in first lactation cows assessed at breeding cattle auctions. Vet. J. 183:161-165.

Jones, G., W. O. Johnson, W. D. Vink, and N. French. 2012. A framework for the joint modeling of longitudinal diagnostic outcome data and latent infection status: Application to investigating the temporal relationship between infection and disease. Biometrics 68:371-379

Laven, R. A. 1999. The environment and digital dermatitis. Cattle Pract. 7:349-354.

Laven, R. A., and D. N. Logue. 2007. The effect of pre-calving environment on the development of digital dermatitis in first lactation heifers. Vet. J. 174:310-315.

Lunn, D., D. Spiegelhalter, A. Thomas, and N. Best. 2009. The BUGS project: Evolution, critique, and future directions. Stat. Med. 28:3049-3067.

Miklossy, J., S. Kasas, A. D. Zurn, S. McCall, S. Yu, and P. L. McGeer. 2008. Persisting atypical and cystic forms of Borrelia burgdorferi and local inflammation in Lyme neuroborreliosis. J. Neuroinflammation 5:40.

Moe, K. K., T. Yano, K. Misumi, C. Kubota, W. Yamazaki, M. Muguruma, and N. Misawa. 2010. Analysis of the IgG immune response to Treponema phagedenis-like spirochetes in individual dairy cattle with papillomatous digital dermatitis. Clin. Vaccine Immunol. $17: 376-383$

R Core Team. 2013. R: A language and environment for statistical computing. R Foundation for Statistical Computing, Vienna, Austria.

Rasmussen, M., N. Capion, K. Klitgaard, T. Rogdo, T. Fjeldaas, M. Boye, and T. K. Jensen. 2012. Bovine digital dermatitis: Possible pathogenic consortium consisting of Dichelobacter nodosus and multiple Treponema species. Vet. Microbiol. 160:151-161.

Read, D. H., and R. L. Walker. 1998. Papillomatous digital dermatitis (footwarts) in California dairy cattle: Clinical and gross pathologic findings. J. Vet. Diagn. Invest. 10:67-76.

Relun, A., R. Guatteo, P. Roussel, and N. Bareille. 2011. A simple method to score digital dermatitis in dairy cows in the milking parlor. J. Dairy Sci. 94:5424-5434.

Relun, A., A. Lehebel, M. Bruggink, N. Bareille, and R. Guatteo. 2013 Estimation of the relative impact of treatment and herd management practices on prevention of digital dermatitis in French dairy herds. Prev. Vet. Med. 110:558-562.

Santos, T. M. A., R. V. Pereira, L. S. Caixeta, C. L. Guard, and R. C Bicalho. 2012. Microbial diversity in bovine papillomatous digital dermatitis in Holstein dairy cows from upstate New York. FEMS Microbiol. Ecol. 79:518-529.

Stokes, J. E., K. A. Leach, D. C. J. Main, and H. R. Whay. 2012. The reliability of detecting digital dermatitis in the milking parlour. Vet. J. 193:679-684. 
Trott, D. J., M. R. Moeller, R. L. Zuerner, J. P. Goff, W. R. Waters, D. P. Alt, R. L. Walker, and M. J. Wannemuehler. 2003. Characterization of Treponema phagedenis-like spirochetes isolated from papillomatous digital dermatitis lesions in dairy cattle. J. Clin. Microbiol. 41:2522-2529.

Umemoto, T., I. Namikawa, and M. Yamamoto. 1984. Colonial morphology of treponemes observed by electron-microscopy. Microbiol. Immunol. 28:11-22.

Vink, W. D., G. Jones, W. O. Johnson, J. Brown, I. Demirkan, S. D. Carter, and N. P. French. 2009. Diagnostic assessment without cut-offs: Application of serology for the modelling of bovine digital dermatitis infection. Prev. Vet. Med. 92:235-248.

Walker, R. L., D. H. Read, K. J. Loretz, D. W. Hird, and S. L. Berry. 1997. Humoral response of dairy cattle to spirochetes isolated from papillomatous digital dermatitis lesions. Am. J. Vet. Res. 58:744-748.

Walker, R. L., D. H. Read, K. J. Loretz, and R. W. Nordhausen. 1995. Spirochetes isolated from dairy cattle with papillomatous digital dermatitis and interdigital dermatitis. Vet. Microbiol. 47:343-355. 